Aus der Heilstätte Heidehaus bei Hannover. (Chefarzt: Dr. Ziegler.)

\section{Orthodiagraphische Untersuchungen über die Herzgröße bei Tuberkulösen.}

Von cand. med. Paul Kersten (Kaiser Wilhelms-Akademie.)

Die Frage nach der Helzgröße bei Tuberkulösen ist schon vielfach erörtert worden. Von den sich in der Hauptsache gegenüberstehonden Ansichtelı geht die cine dahin, daß das Herz bei vielen Phthisikem von vornherein kleiner sei, als es normalerweise sein müßte, d. h. daß das Verhältnis zwischen Körpergröße und Köı pergewicht einerseits ınd Helzvolımen anderseits schon vor Beginn der tuberkulösen Erkrankung verändert sei im Sinne einel Verringelung des Helzvolumens und da $\beta$ man in diesem Mißverhältnisse einen wichtigen Faktor fïr die Dispcsition zur Tuberkulose finden könnc. Von andeler Seitc wird das kleine Hel'z, wenn man es wiıklich findet, nur als Synıptom von solchen vorgeschrittenen Eikrankungen angesehen, mit denen eine allgemeine Kachexie einhergeht, dagegen ein prinü̈r kleines $\mathrm{Helz}$ in Abrede gestellt.

Schon Rokitansky hat auf eine auffällige Organisation des Körpers hingewiesen, dic zur Tuberkelbildung in den Lungen disponiere, ausgezeichnet durch ,große Thoraxräune neben Kleiıhcit des Herzens und zartem Bau der Gefäße."

Der hauptsächlichste Verfechter der Theorie ron primär kleinen Herzen ist Breli mer gewesen. Er betont als besonders charakteristisch für einen großen Teil Tuberkulöser dies angefülırte Vïßverlı̈lthnis zwischıen Herz und Lungen. Dabei brauchten die Kranken niclit so ohne weiteres das Bild des paralytischen Thora $\mathbf{x}$ darzubieten, man finde diesen sogar bei beginnender Tuberkulose keineswegs im Uebernıß, sondern gerade das Mïßverhältnis zwischen Herz und Lunge, niclıt die äußere Thorax form sei die morphologische Eigentümliclikeit des Habitus phtlisicus. So hält denn Brelı mer auch die Kleinheit des Herzens und Anonıalien im Gefäßsystem für eines der hauptsächlichsten zur' Tuberkulose dis. ponierenden Niomente; denn ein solches kleines Herz könne auch nur eine geringere als die normale Blutmenge aufnehmen und köune auch nur eine geringere bei jeder Kontraktion an den Köıper, resp. all seinc einzelnen Organe abgeben, und dumit sei die Elnälırung keine normale. Als plıysiologische Folge werde in den Lungen ein Locus nimoris resistentiae geschaffen, und folglich könnten notwendigerweise die T'uberkelbazillen dann gerade in den Lungen ihre Wirkung entfalten. In der Tat, ein so kapillarenreiches Gewebe wie die Lunge bedarf eincr beträchtlichen Blutversorgung, und bei eiıenı verhältnisınäßig zu kleiıen Herzen muß diese durch dic geringere als normale Blutmasse, die durch den rechten Ventrikel lierauggeworfen werden kann, ein verluältnisnı̈̈Big langsames Strömen des Blutes bewirken. Jedoch wird mit Recht, wie es scheint, der Einwand gellacht, daß man nicht ohne weiteres aus ciner anatomischen Beschaffenheit unbedingt sichere Schlüsse auf die Funktion zielıen dürfte. Denn eine Herzkanınıer leistet nicht immer erhölıte Arbeit proportional der Dicke ihrer Wandungen. Es kann cinc zur Phthise disponierende nuangelnde Durchblutung der Lungen mindestens ebensogut einem funktionell sclwachen wie kleinen Herzen zur Last gelegt werden. Nach Kraus ist das kleine Herz der Individuen mit engbrüstigem Habitus auch konstitutioneil schwach.

Neben Brelımer ist es hauptsächlich Beneke, der für das primär kleine Herz eintritt. Er weist noch außerdem darauf linı, da B gerade in der Pubertätszeit das Herz seine hauptsächlichste Fntwicklung erfahre, oft aber zurückbleibe, und daß dann im Vergleich zu delll übrigen schnellen Wachstumı ein Mißverhältnis zwischen Herz und übrigem Körper die Folge sei. Naclı Ortlı gelı̈ren vol allenı bei den Fällenı, die in der Pubertätszeit zu Tode kämen, die enge Aorta und das kleine Herz zu ganz gewölnnlichen Erscheinungen. Die Frage, ob diese Tatsache mit der Disposition in Zusammenhang zu bringen sei, läßt er offen.

Im Gegensatz zu diescr Ansiclıt vom prinıär kleinen "Herzen stelıt dic oben erwähnte voln sekundär kleinen, atrophischen Herzen. Nian köıne, meint Colınlıeim, die ungleiche Widerstandsfähigkcit gegeı die Ausbreitung des Virus als eincn Ausfluß der Konstitution des Individuunıs ansehen, dürfe diese nicht mit dcu zusanmmenwerfen, was man unter phthisischem Habitus, also kleinem Herzen etc. verstehe, da dieser mit der Empfänglichkeit für Tuberkulose niclıts zu tun habe, sondern lediglich ein Produkt der Krankheit sei. Fbeuso Cornet. Henle, die meinen, der Habitus phthisicus sei inmuer schon ein Symptonı der Krankheit, die Kleinlıeit des Herzens sei keine Hypoplasie. sondern gehe lediglich der Abmagerung des gesamten Körpers, speziell der Miuskulatur parallel. Daher hättcn dic am stärksten abgenıgerten Kranken auch die kleinsten Herzen. Andere Autoren nehmen eine Mittelstellung ein: Rokitansky, Salıli, Licbermeister u. a.

Man sieht also, daß es ans der Literatur schwierig ist, Stellung zu der Frage zu nehmen; denn zahlenmäßige Auf- stellıngen sind kaun vorhanden, und die wirklich volhandenen stammen größtenteils aus klinischen Eindrïcken und sind infolgedessen mehr oder woniger subjektiv. Objektive Beobachtungen konnte man nur bei der Sektion machen, und die Falle von beginnender Tuberkulose kommen selten auf den Sektionstisch. Damit ist das Fehlen statistischer Angaben ïber die Herzoröße bei beginnender Tuberkulose ver'ständlich.

Ein wichtiges Mittel, das Problem der_Lösung näher zu bringen, ist die Untersuchung mittels der Orthodiagraphie. Ueber die Zuverlässigkeit der Methode sind von Moritz sorgfältige Untersuchungen gemacht worden. Allerdings muß eingestanden werden, da 3 die Ansmessung eines Orthodiagramms nicht völlig objektiv ausg efüh't werden kann. Die rechte und linke Grenze ist zwar bei jeder Aufnahme gegeben, aber nicht die untere und obere. Es bleibt daher kein andercr Answeg, als genüß dem Vorschlag von Morit z die fehlenden Stellen nach Lage der nächstgelegenen Punkte zu ergänzen. So wenig es anch scheint, so sind nach den praktischen Elfahrungen die Fehler dabei gering.

Die von mil ausgefiihrten Untersuchungen wurden auf dem horizontal gestcllten Orthodiagraphen vorgenommen. Gemessen wurde der Längs-, Quer- ind del transversale Durchmesser. Die gewonnenen Resultate wurden mit den von Dietlen berechncten Mittelzahlen verglichen. Es sei bemerkt, daß bei allen Fällen, die in der Statistik verwertet worden sind, die Tuber kulose entweder durch den Nachweis von Tuberkelbazillen im Auswurf oder durch positive Tubel kulinreaktion neben den sonstigen anskultatorischen, perkutorischen und löntgenographischen Befunde sichergestellt ist. Fälle, bei denen ein Zweifel bestand, sind unberiicksichtigt geblieben. In Fragc kommen 129 Fälle, die siclı auf alle drei Stadien der Tuberkulose erstrecken (Turban-Gerhard tsche Einteilung)

Da anzunehmen ist, daß körperlich schlecht ernährte ind zart gebaute Individuen von vornherein ein kleineres Hierz haben als kräftige, wurde eine Trennung vorgenommen, und zwar. wurde das ganze Material in zwei Hauptgruppen eingeteilt: in normal und sehlecht Genälıte. Es ergab sich folgendes tabcllarische Bild:

Es hatten von:

A. 104. noinzal Eruährten

I. $65:=62,5 \%$ zu kleine Herzen. II. $39=37,5 \%$ nor'nale Her'zen, vom l. Stadium 20
24
3. $\quad \cdots \quad 21$

vom 1. Stadium 15

B. "25 schlecht. Erruälirten

$\begin{aligned} \text { I. } 22=88 \% \text { zu kleine } & =8 \\ & \text { von } 1 \text {. Stadiun } 5\end{aligned}$

$$
3 .
$$

11. $3:=12 \%$ nomale Herzen, vom 1. Stadiun 1

$$
2 . \quad \cdots \quad 2
$$

Fiir die Beurteilung unserer Frage sind besonders die unter A I gesetzten 65 normal ernährten Kranken, bei denen Verkleinerungen bis zu Zweidrittel der relativ normalen Herzsilhouette vorkamen, wichtig, dazu teilweise bei erst ganz kurze Zeit bestehendcr Erkrankıng. Die 21 Fülle von diesen 65, die dem Lungenbefunde nach ins dritte Stadiunı eingereiht wurden, boten trotz ihrel schweren Erkrakkung absolut nicht das Bild eincs abgemagerten Körperzustandes. Also kann auch hicl die Kleinheit nicht als eine Folge von allgemeiner Atrophie angesehen werden.

Von den 25 schlecht Eınährteı hatten 22 zu kleine Herzeı; lier muß man die Frage, ob die Kleinheit als primäre oder sekundäre Lrschcinung anzusehen ist, offen lassen. Man darf aber vel'muten, daß ein Teil, und zwal del größcre, schon inı Beginn der Phthise klein gewesen ist. Es wird also die Frage, ob das Herz bei Tuberkulösen primär klein oder sekındär atrophisch ist, fïr viele Fälle, und zwar dem weitans größeren Teil, im Sinne Brelımers und Benekes beantwortet.

Inwicfern wirklich das kleine Herz von Bedeutung bei der Disposition werden kann, muß zukiinftigen Forselungen ïberlassen bleiben. So einlenchtend es anf den ersten Blick zu seiı schien, daß bei den engen Beziehungen zwischen Respirationsund Zirkulationsapparat eine Schädigung der Triebkraft des Herzens nicht ohne scliädlichen Einfluß auf den Ernährungszustand und die Funktion der Lunge bleiben kann, so ist doch eine allseitige Beurteilıng dieser Verhältnisse schwierig. Die 
wesentlichste Rolle spielt nach K r e h I die Strömungsgeschwindigkeit des Blutes. Man kann diese, sofern die Weite der GefäBe dort nur geringen Schwankungen unterliegt, direkt parallel setzen den Blutmengen, welche das Organ in der Zeiteinheit durchströmen, und diese wiederum hängen von der Druckdifferenz zwischen Lungenarterie und linkem Vorhof ab. Bei den geringen Druckunterschieden kann, wenn eine gewisse Geschwindigkeit nicht eingehalten wird, das Entweichen der Kohlensäure als auch das Eintreten des Sauerstoffs in das Blut empfindlich gestört werden. Und wenn man wie Boh r Drüsenkräfte für wirksam hält, muß man jedenfalls der Schnelligkeit des Blutfließens eine große Bedeutung beilegen, denn von dieser sind alle Drüsenfunktionen im hohen Maße abhängig. Angesichts dieser Bedeutung der Stromgeschwindigkeit ist eine Schädigung der Ernährung und Funktion des Lungengewebes durch Schwächezustände des Herzens verständlich. Bekräftigt wird diese Ansicht durch die alte Erfahrung, da 3 Personen mit Stenose der Iungenarterie bzw. des Ostium pulmonale häufig phthisisch werden. -

Nicht zu verwechseln mit dem besprochenen Langsamströmen des Blutes in der Lunge ist die durch manche Klappenfehler (Mitralfehler und Aortenklappenfehler) hervorgerufene Stauung in den Lungen, die im Gegenteil als zum mindesten erschwerend, wenn nicht gar ausschließend für die Ausbreitung der Tuberkulose in den Lungen angesehen wird.

Zusammenfassung. Die Frage nach der Herzgröße bei Lungentuberkulose wird verschieden beantwortet. Eine Ansicht geht dahin, daß das Herz bei Tuberkulösen größtenteils primär, d. b. vor oder bei dem Ausbruch der Krankheit zu klein sei im Verhältnis zu Körpergröße und Gewicht, und man glaubt darin ein wichtiges Moment für die Disposition sehen zu können. Andere sehen die Kleinheit, wenn man sie wirklich beobachtete, als eine Atrophie an, die mit der Abmagerung des übrigen Körpers einhergeht. Orthodiagraphische Untersuchungen ergeben auch bei eben beginnenden leichten Erkrankungen und auch bei fortgeschrittenen mit gutem Ernährungszustande einen großen Prozentsatz erheblich zu kleiner Herzen, sodaß man dies auffällige Symptom für die Disposition in Betracht ziehen muß, wie es Brehmer und Beneke schon getan haben.

Die Frage, in welcher Weise ein kleines Herz Schädigungen in den Lungen herbeiführen kann, muß unentschieden gelassen werden. 